\title{
Student understanding of measurement and uncertainty: probing the mean
}

\author{
Nuraan Majiet $^{1}$ and Saalih Allie ${ }^{1}$ \\ ${ }^{1}$ Department of Physics, University of Cape Town, Rondebosch, South Africa, 7701
}

\begin{abstract}
The present work is part of a broad programme aimed at understanding how students think about data obtained from measurement, including measurement uncertainty, at a more fine-grained level than that obtained from the Physics Measurement Questionnaire. More specifically, to what extent do measured student shifts from a point to a set paradigm come about due to actual conceptual change or are they the result of recognizing familiar situations that can successfully be processed according to appropriate prescriptions. In order to probe these finer grained aspects of understanding a process of developing a suitable questionnaire was initiated. The first phase of the process involved piloting individual, specific questions. The present paper will discuss the questions that were developed and piloted with a group of students after an introductory lab course. The focus of the paper will be on the level of informativeness of the responses that were obtained.
\end{abstract}

\section{INTRODUCTION}

Studies probing student understanding of measurement and uncertainty were carried out over several years by the Department of Physics at the University of Cape Town in collaboration with the Department of Educational Studies at the University of York. The work commenced in 1998 with a paper [1] that included a description of an instrument, that had been developed to explore student understanding with regard to various concepts that were associated with taking measurements and analyzing data. A key insight from the work was the observation that all student responses could be ascribed to two fundamental ways of engaging with data, the Point and Set paradigms [2]. The goal of teaching in this area was then to shift students from the former to the latter as measured in pre- and post-tests. A more recent version of the instrument, now known and the Physics Measurement Questionnaire (PMQ), is described in ref [3]. This reference also details the Point as Set paradigms and shows how a particular teaching approach facilitated a successful transition from Point to Set for most students in a physics major class.

However, while the PMQ is informative in terms of measuring the pre- and post-test shifts, it is not designed to establish why the paradigm shift occurred. The present work is an attempt to try and understand why the shift occurred. In other words, do shifts from a Point to a Set paradigm result from deep conceptual change or are they the result of recognizing familiar situations that can successfully be processed according to appropriate prescriptions? In order to probe these finer grained aspects, a process of constructing a suitable instrument is presently underway. The first phase of the process has involved piloting various questions. The present paper will discuss the questions that were developed and piloted with a group of students after an introductory lab course. The focus of the paper will be on the level of informativeness of the responses that were obtained, and thereby to accept, modify or reject each question as suitable for the overall instrument.

\section{METHODOLOGY}

\section{A. Development of the Questionnaire}

Our previous studies have shown that both the posited context and question format have significant influences on how respondents answer the questions. This is related to issues around priming and framing. Thus, in order to be able to compare and relate the results from the pilot study to previous work we constructed the new instrument to resemble the overall form of the PMQ. Hence we used the same posited context and the first two questions of the PMQ as the opening questions of the pilot instrument.

The final version of the pilot instrument consisted of six written questions. The first two questions which were copied from the PMQ related to data collection (Repeating Distance (Q1) and Repeating Distance Again (Q2)) [3].

The four new questions (Q3-Q6) all focused on the respondents' views of data processing. Three of these questions specifically dealt with the respondents' interpretation of the mean. Question three investigated the respondents' thought process in using the mean as the final result in an experiment. Question four investigated the respondents' use of the mean to predict the outcomes of future data whilst question five investigated the respondents' use of the mean in calculations. In each of the questions the mode or recurring value was introduced as an alternate measure of central tendency. The final question looked at the respondents' decision process when combining data graphically. This question will not be discussed further. 


\section{B. Framing the question}

All questions were framed in the form of a discussion where three or four posited students debated the nature of various laboratory procedures and data analysis techniques. The posited students were represented by genderless, raceless cartoons to avoid bias [2]. The respondents were required to select an opinion with which they most closely agree. The respondents were required to give a tick-a-box response followed by a qualitative description explaining why they made that choice.

\section{Student sample}

The questionnaire was administered to a sample of first year students at the University of Cape Town. The respondents were all in an extended curriculum introductory physics course (PHY1023H) after being advised to transfer from one of two mainstream physics courses after a placement test.

PHY1023H was designed at providing students with the skills, tools and conceptual understanding necessary for engaging in a full first year physics course.

[4]. The content is covered over one and a half years and has the same credits as a semester course.

\section{Administering the questionnaire}

The questionnaire was administered to 98 non-majoring physics students at the end of the second semester in 2017. Therefore, these students would have attended and completed 8 practical sessions before completing the questionnaire.

The respondents were informed that the data would be analysed anonymously and that their lecturer would not be able to identify them by their responses. The students were informed that the questionnaire was being administered for research purposes.

The questionnaire was administered online. It was conducted during a tutorial session to maximize student participation, to ensure that all respondents had internet access as well as to ensure that the respondents completed the questionnaire individually/independently. Due to protest action at the University of Cape Town in the name of the fees must fall movement [5], the students were interrupted while completing the questionnaire. Therefore, the present work yielded 20 full sets of completed responses.

\section{ANALYSIS AND RESULTS}

In previous exploratory studies conducted by us, analysis of post-instruction questionnaires or questionnaires which investigate conceptually difficult areas in physics or astronomy has shown to yield data that are not very informative i.e. inappropriate for a grounded analysis approach [6-7]. Therefore, a new preliminary analysis technique was piloted and used to determine whether the developed questionnaire was successful at yielding data of a high level of informativeness. This technique involved ranking responses according to the level of informativeness.
Correctness with the theory did not form part of the criteria when ranking responses. Therefore, the categorization was mainly based on the clarity and elaboration of the ideas expressed by the respondents.

TABLE I. Coding Scheme.

\begin{tabular}{cl}
\hline $\begin{array}{c}\text { Informativeness } \\
\text { Level }\end{array}$ & Criteria \\
\hline Low (LI1) & No explanatory idea \\
Medium (LI2) & $\begin{array}{l}\text { Explanatory ideas with little } \\
\text { elaboration }\end{array}$ \\
High (LI3) & $\begin{array}{l}\text { Explanatory ideas with } \\
\text { considerable elaboration }\end{array}$ \\
\hline
\end{tabular}

The final version of the pilot questions can be found in the Appendix.

Below are examples of responses for each category arranged according to the question which prompted the response. The responses were quoted without editing but in some instances inferred words were placed in brackets to provide clarity.

\section{A. The mean as the final result [MFR]}

Question 3 was the first question that varied from the PMQ. It investigated the respondents' views of data processing, in particular the use of the mean as the final result in an experiment as well as the interpretation of variation in a repeated measurement experiment. The variation in a data set was framed as "right or wrong" results.

An example of a level 1 response:

- "The mean will give us an average value of all the readings."

An example of a level 2 response:

- "The mean provides a better best approximation than the each measurement from the experiment that would have some uncertainty."

An example of a level 3 response:

- "These results are due to the experiment not being in an isolated system, therefore many factors can affect the experiment and therefore yield different results neither wrong nor right. Only an isolated system will yield exact results matching to theory."

\section{B. Predicting repeated distance measurement [PR]}

Question 4 investigated the respondents' views about predicting the outcome of future data after obtaining several repeated measurements. The question was framed in the context of a bet.

An example of a level 1 response:

- "We will choose the value that appears the most." 
An example of a level 2 response:

- "Another reading will slightly change the mean value and definitely won't get the number that was mostly repeated."

An example of a level 3 response:

- "The mean and the most achieved value are important, but they won't happen all the time. What will happen is that the ball should land somewhere within the uncertainty, or a few uncertainties of the mean."

\section{Using repeated distance measurement [UR2]}

Question 5 investigated student views on what to use of the final result of a data set to perform calculations. An example of a level 1 response:

- "that value (mode) is very likely to be correct"

An example of a level 2 response:

- "The mean value makes more sense since it represents widely spread data and even takes care of the odd readings (outliers)"

An example of a level 3 response:

- "The mean value gives an approximation of the true distance in which the ball lands. Taking the mode or the repeated number would leave out other readings and would assume that the reading is the true distance landed while it has an uncertainty the same as the rest of the results collected."

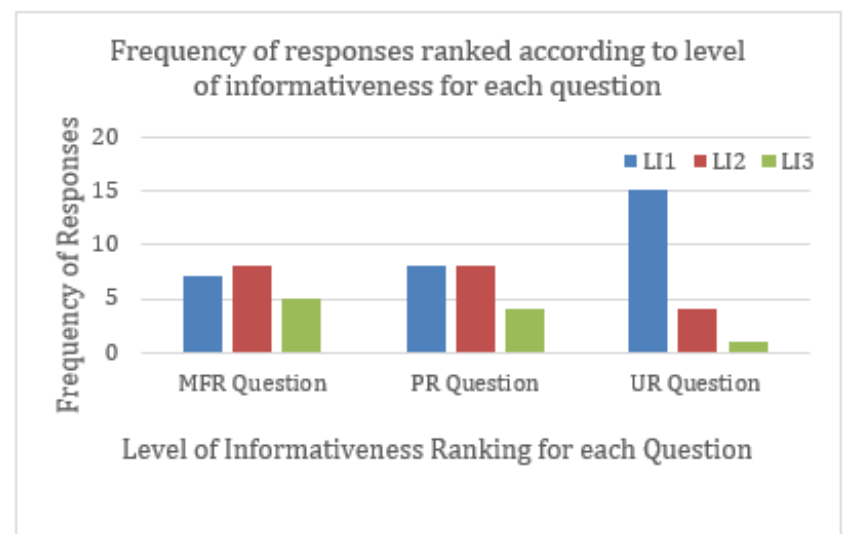

FIG 1. A graph of the frequency of responses ranked according to level of informativeness for each question. There was a total of 20 responses for each question. More than $50 \%$ of the responses were ranked as a level 2 or lower for each question.

The majority of the respondents provided the same measure of central tendency for questions 3 and 5 while a few respondents switched from the mean to the mode when attempting to predict future data in question 4 . Most of the data with a high level of informativeness came from the responses in which the respondent selected option C; the alternate option. Most of the level 1 data consisted of definitions or jargon, for example; "the mean is the center of location", "the mean is the most certain value" and "the mean is more accurate", while the level 2 and 3 data were a combination of definitions and further explanations.

The first two questions of the pilot questionnaire indicated that more than $50 \%$ of the respondents used the expression "average" to motivate why they wanted to repeat measurements. Previous studies have shown that before instruction this term is used loosely by respondents and can have array of student interpretations [2]. Findings for the present study indicate that the respondents still do not have a well-established understanding of the mean after instruction. A few case studies are highlighted below:

- "Because it (the mean) is the most middle number and hence the most reliable one."

- "Using the mean provides a better best approximation because its in the middle of the interval and it (is) likely to be close to the actual value."

- "I'm not sure why we take a mean."

It should be noted that while the first two responses quoted above may hold for a symmetrically distributed data set, the one provided was skewed and therefore a simple calculation made by the respondent would have shown them that the use of the mean as the best result and their motivation provided are not in agreement.

There also appears to be a limited understanding amongst respondents about the relationship between the mean (a discrete value) and the variation (represented by a distribution/interval) in a data set:

- $\quad$ "The mean gives the average of the results, which is the value that we are most interested in. It shows how close our results are to each other.

- "The mean will give a rough estimate of the correct answer and if uncertainty is taken into account it will give a much closer value to the true value."

Keeping this in mind the following responses are ambiguous to interpret.

- "The mean value makes more sense since it represents widely spread data and even takes care of the odd readings (outliers)."

- "By calculating the mean, you are only decreasing the uncertainty."

\section{CONCLUSIONS}

The pilot questionnaire gave rise to data that mainly categorized as having a low level of informativeness as more than $50 \%$ of the responses ranked as a level 2 or lower. Many of the responses were ambiguous and made it difficult to infer the level of student understanding of the nature of the mean.

From what could be inferred, respondents appeared to have a limited view of the mean as the best approximation in a repeated measurement experiment. The findings suggest that the shift has indeed occurred due to the result of recognizing familiar situations that can successfully be processed according to appropriate prescriptions, but due to 
the low level of informativeness of the data obtained, it still remains largely unclear.

Question 5 provided the least informative responses and suggests to us that it be omitted in future questionnaires. Of consideration is also for the questionnaire be administered in written format so as to encourage students to include diagrams in their explanations [8-9].

Despite the small sample size, the nature of the responses serves as a guideline for our future work, in particular with regard to the formulation of future instruments.

\section{ACKNOWLEDGEMENTS}

We would like to thank the University of Cape Town and the Academic Development Programme (ADP) for funding this research.

\section{APPENDIX}

QUESTION 3: Mean as the final result

The students continue to release the ball down the slope at a height $h=400 \mathrm{~mm}$.

They obtain the following after six release:

$\begin{array}{rr}\text { Release } & d(\mathrm{~mm}) \\ 1 & 436 \\ 2 & 425 \\ 3 & 440 \\ 4 & 425 \\ 5 & 434 \\ 6 & 425\end{array}$

One of the students says, "Great. We should now calculate the mean as the final result."
The following discussion then takes place between the students.

A: We can't take the mean! You can't combine results that are right and wrong.

B: It doesn't matter if some are right or some are wrong. The mean takes care of it.

C: It isn't about right or wrong. Let me explain what is going on.

QUESTION 4: Predicting Repeated Distance Measurement:

The students take a bet on what result they will get for $d$ if they release the ball again at a height $h=400 \mathrm{~mm}$.

The following discussion then takes place between the students.

A: We have practiced a lot so I bet we will get the mean value.

B: No way! We will get the value that was repeated the most.

C: You are both wrong! Let me tell you what will really happen and why.

QUESTION 5: Using repeated distance measurement: The students then discuss what value to use for $d$ in an equation.

A: We have to use the mean value.

B: We have to use the value that repeated itself the most.

C: I don't think you understand what is going on. Let me D: explain.
[1] S. Allie, A. Buffler, B. Campbell, \& F. Lubben, Firstyear physics students' perceptions of the quality of experimental measurements. International Journal of Science Education, 20(4), 447-459. (1998)

[2] B. Campbell, F. Lubben, A. Buffler, \& S. Allie, Monograph, Teaching scientific measurement at university: Understanding student ideas and laboratory curriculum reform, p.73, (2005).

[3] T. Volkwyn, S. Allie, A. Buffler, \& F. Lubben Impact of a conventional introductory laboratory course on the understanding of measurement. Physical Review Special Topics - Physics Education Research, (2008).

[4] Faculty of science handbook, University of Cape Town. (2018).

[5] L. Hauser, 'Fees Must Fall': Anatomy of the Student Protests in South Africa. The New York Times, (2016).
[6] J. Bok, unpublished honors project; Probing student views about the Big Bang in an introductory astronomy course. University of Cape Town, (2014).

[7] N. Majiet, unpublished honors project; Student views on the role of experiments in physics, University of Cape Town, (2016).

[8] T. Bartiromo, J. Finley, E. Etkina, Searching for Evidence of Student Understanding. Paper presented at the Physics Education Research Conference, (2010).

[9] A. Saalih \& D. Damaree, Toward Meaning and Scientific Thinking in the Traditional Freshman Laboratory: Opening the "Idea Space", Paper presented at the Physics Education Research Conference, (2010). 